Ann. Abeille, $\mathrm{I}_{964}, 7(\mathrm{r}), 55^{-6 \mathrm{r}}$.

\title{
LA CLAUSTRATION DES MÂLES DESTINÉS A L'INSÉMINATION ARTIFICIELLE DES REINES D'ABEILLES
}

\author{
J. FRESNAYE \\ Station expérimentale d'Apicullure, \\ Centre de Recherches agronomiques du Sud-Est, Montfavet (Vaucluse)
}

SOMMAIRE

La claustration des mâles d'abeilles est indispensable lors de travaux de sélection ou de génétique faisant appel à l'insémination artificielle des reines. L'auteur propose deux types de cages, l'une de très petit volume, fixée devant la ruche, utilisable dans les ruchers éloignés; l'autre dans laquelle on peut entrer pour effectuer les visites de la ruche. Dans les deux cas, les abeilles conservent une activité normale et rapportent miel, pollen et eau à la ruche. Les mâles volent dans les cages et atteignent une parfaite maturité. Ils retrouvent la ruche plus facilement que les abeilles ouvrières, ce qui est sans doute en rapport avec le développement bien connu de leurs facultés visuelles et olfactives.

\section{INTRODUCTION}

On sait depuis longtemps que les mâles d'Apis mellifica ou faux-Bourdons changent facilement de ruche et GoETzE (I955) a démontré que cette " dérive " pouvait atteindre $50 \mathrm{p}$. Ioo de la population mâle d'une colonie. Cependant, pour la rigueur des programmes de sélection et des recherches concernant la génétique de l'abeille, il est indispensable d'être informé sans erreur possible de la filiation des mâles aussi bien que de celles des reines auxquelles ils s'accouplent. S'il est facile de s'assurer de l'origine des reines qui peuvent être marquées et claustrées dès leur naissance, par contre, l'identification des mâles est plus délicate. Le seul moyen pratique de s'assurer de leur origine reste la claustration.

La méthode de claustration des mâles la plus répandue est décrite par LAIDLAW (I954) et utilisée pour l'insémination artificielle des reines. Les mâles sont enfermés dès leur naissance dans de petites cages à l'intérieur de la ruche jusqu'à leur majorité. Ils sont ensuite placés dans une " boîte de vol " juste avant leur utilisation 
pour l'insémination artificielle. Ils vivent très bien dans ces cages, car les abeilles pénètrent à l'intérieur pour les nourrir. Ils ne peuvent cependant pas voler et leur maturation peut en être affectée. D'autre part, les manipulations sont complexes, surtout lorsqu'il faut claustrer plusieurs milliers de mâles de diverses origines. Nous avons donc cherché à améliorer la technique de claustration des mâles et nous présentons ici le résultat de nos essais.

\section{MATÉRIEI,}

Nous avons construit plusieurs types de cages afin d'étudier le comportement des mâles dans différents volumes. Toutes ces cages étaient munies d'un dispositif permettant aux abeilles de sortir à l'extérieur. Les colonies pouvaient ainsi subvenir à leurs besoins en miel, pollen et eau sans notre intervention.

\section{1) Ruche placée à l'intérieur d'une petite cage}

La cage est constituée par des cadres de bois, grillagés, faciles à assembler (fig. I). Ses dimensions extérieures sont de $\mathrm{I}, 80 \mathrm{~m}$ de haut, $\mathrm{I}, \mathrm{IO} \mathrm{m}$ de large et $0,85 \mathrm{~m}$ de profondeur. Ces mesures représentent le volume minimum nécessaire pour qu'un opérateur puisse y travailler à l’aise. Un dispositif placé à l'entrée de la ruche (fig. I et $2-\mathrm{A}$ ) de $0,35 \mathrm{~m}$ de long sur $0,37 \mathrm{~m}$ de large et $0,03 \mathrm{~m}$ de haut, fait communiquer directement la ruche avec l'extérieur de la cage. La colonie garde son activité normale; les abeilles vont et viennent, mais les mâles sont retenus par une grille type "grille à reine " (fig. $2-A$ ). Sur le côté de cette entrée de ruche, à l'intérieur de la cage, une ouverture est ménagée pour permettre aux mâles de voler. Une planche de vol y est accolée ; elle leur assure un meilleur repérage visuel et olfactif (fig. $2-B$ ). Cet orifice est fermé pendant quelques jours, au début de l'installation de la ruche dans la cage, pour que les abeilles s'habituent à utiliser l'entrée principale qui leur est destinée. Le toit est opaque, ainsi que le haut de trois parois sur une hauteur de $0,30 \mathrm{~m}$ (fig. I - B). Les quelques abeilles qui pénètrent dans la cage lorsque l'ouverture pour les mâles est ouverte s'éloignent de cette zone plus sombre pour s'approcher de la paroi avant qui est plus claire et dont la partie haute est munie d'une grille à reines leur permettant de sortir de la cage (C). La partie basse de la paroi (D) se sépare au besoin du reste de la cage pour permettre l'installation plus facile de la cage autour de la ruche. Une paroi (E) sert de porte pour pénétrer dans la cage afin d'y effectuer les prélèvements des mâles et les visites.

\section{2) Ruche placée à l'intérieur d'une grande cage}

La grande cage a pour dimensions extérieures $2 \mathrm{~m}$ de hauteur, 2,10 $\mathrm{m}$ de longueur et $\mathrm{I} \mathrm{m}$ de largeur. Son volume est donc d'environ le triple de celui du modèle décrit ci-dessus. Le dessus est simplement grillagé et non opaque. L'entrée de la ruche est du même type que celle utilisée précédemment.

\section{3) Petite cage fixée devant la ruclie}

Cette cage est de faible dimension : $0,27 \mathrm{~m}$ de hauteur, $0,37 \mathrm{~m}$ de largeur et $0,24 \mathrm{~m}$ de profondeur (fig. 3). Flle est fixée devant la ruche à l'aide d'une lanière de caoutchouc. La sortie des abeilles est à la partie inférieure (fig. $4-A$ ). Une ouverture dans le plafond de cette sortie (B) permet le passage des abeilles dans la cage proprement dite (C). Comme dans les cas précédents, les mâles sont retenus par une grille à reine. L'une des parois latérales de la cage est constituée par une toile seulement fixée par le haut et qui peut être soulevée pour prélever les mâles, ouvrir la trappe ou mettre la grille à reine.

\section{RÉSULTATS}

Les trois types de cage ont été expérimentés ensemble. Les facteurs externes sont donc identiques dans tous les cas. Les essais se sont déroulés pendant I mois. 


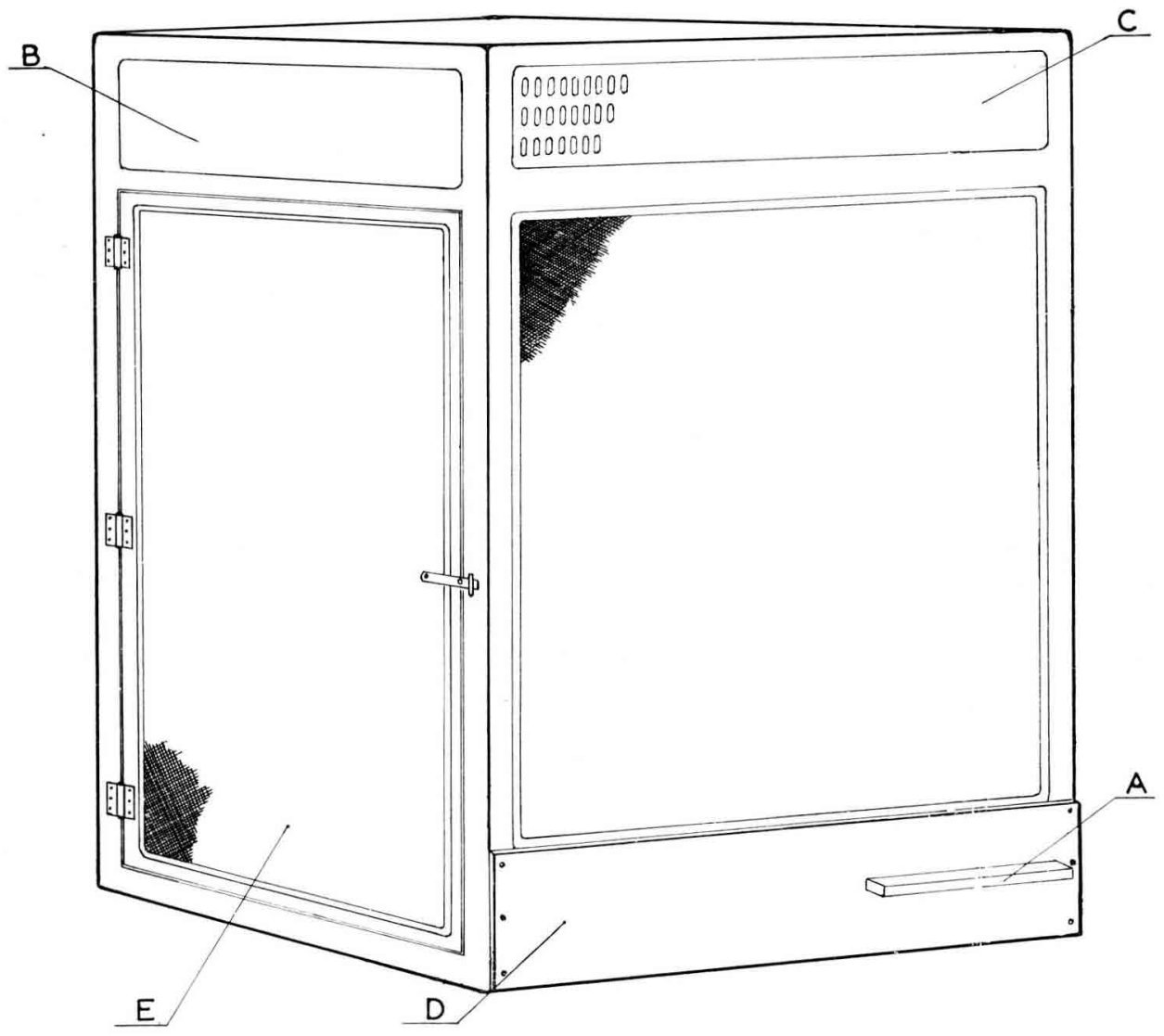

Fig. 1. - La cage avec la ruche à l'intériear

A - Dispositif pour l'entrée et la sortie des abcilles

I3 - Partie opaque

$\mathrm{C}$ - Grille à mâles

D -. Partie amovible

F - Porte grillagé

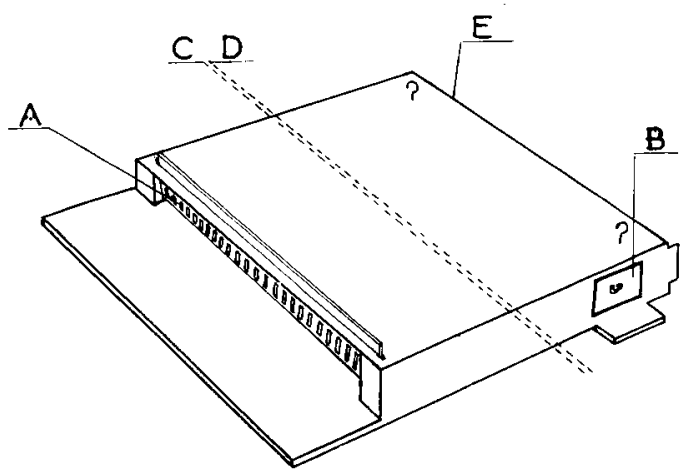

Fig. 2. - Dispositif d'entrée de la ruche

A - Grille à mîles

B - Orifice pour la sortie des mâles dans la cage

C - Partie à l'extérieur de la cage

D - Partie à l'intérieur de la cage

$\mathrm{E} \rightarrow$ Coté fixé à l'entrée de la ruche 


\section{I) Ruche placée à l'intérieur d'une petite caye}

Dans cette cage la mortalité des abeilles est très peu importante. La grande majorité des butineuses utilise la sortie principale qui est adoptée sans difficulté. I,e passage de la grille à reine dont les trous sont rectangulaires est d'ailleurs plus facile pour les abeilles que celui des grilles à pollen dont les trous sont ronds. Parmi les abeilles qui volent dans la cage, et qui sont d'ailleurs assez peu nombreuses, il en est qui retrouvent le passage par lequel elles sont sorties de la ruche; d'autres volent vers le haut de la cage et sortent par la grille à reine antérieure (fig. I - C). I,es mâles volent très bien dans la cage sans se heurter aux parois; ils retrouvent facilement leur entrée de ruche. La mortalité est très faible; on ne retrouve sur le sol que 2 à 3 mâles par jour. Ce nombre particulièrement bas nous incite d'ailleurs à penser qu'il s'agit uniquement de morts naturelles et non d'un épuisement dù à l'encagement. Il semble que ces cages pourraient être plus petites sans inconvénient pour les mâles, mais nous avons déjà signalé que leurs dimensions étaient imposées par la nécessité de pouvoir travailler à l'intérieur. Pour l'insémination artificielle, on prend de préférence les mâles qui volent dans la cage, ce qui évite d'ouvrir la ruche.

\section{2) Ruche placée à l'intérieur d'une grande cage}

I)ans cette cage le comportement des abeilles est conforme à la description de Chacvix (I953). Les abeilles qui se sont égarées dans la cage retrouvent difficilement le trou de vol et elles s'agglutinent en grappes le long du grillage dans les coins de la cage et meurent d'épuisement. Ia mortalité des mâles est faible bien que légèrement plus importante que dans la cage précédente. Ce type de cage présente donc peu d'intérêt puisque la mortalité des mâles et des ouvrières y est supérieure à celles des petites cages et que le montage, le transport et le rangement sont plus difficiles du fait de leur plus grand volume.

\section{3) Petite cage fixée devant la ruche}

Les dimensions de cette cage sont très faibles et il ne se pose, de ce fait, aucun problème de repérage pour les abeilles et les mâles. La mortalité des mâles y est cependant un peu plus élevée que dans les cages précédemment décrites, alors que celle des ouvrières est presque nulle. Il est probable que celles-ci, après avoir tenté vainement de sortir par le grillage, descendent à l'étage inférieur où elles ne rencontrent plus de difficulté pour sortir. Les mâles, par contre, ne disposent que d'un volume trop restreint et leur vol ne s'effectue pratiquement que le long du grillage. En outre, cette méthode a l'inconvénient de nous obliger à enfermer la ruche dans une plus grande cage ou dans une cabine-parasol (FRESNAYE I 964) lors des visites de la ruche, afin d'empêcher les mâles de s'échapper et de se mêler à ceux des autres ruches. Cette petite cage nous est cependant nécessaire; nous en donnerons l'explication en décrivant à présent la méthode de travail.

Les colonies choisies pour fournir des mâles pendant une saison ne sont pas utilisées ensemble et ne peuvent être toutes installées sur les lieux voisins du poste 


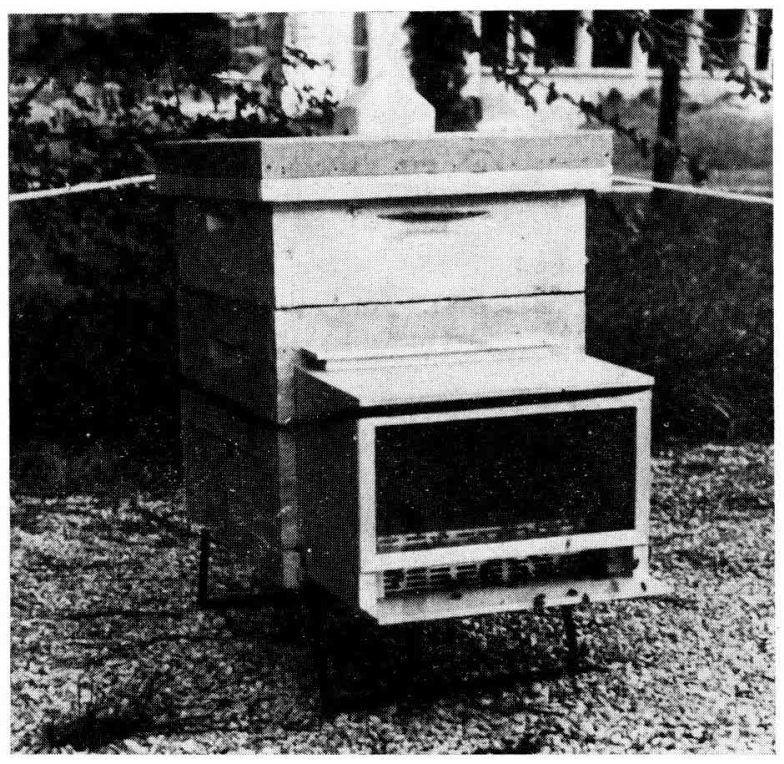

Fig 3. -.- Ia petite cage fivée devant la ruche

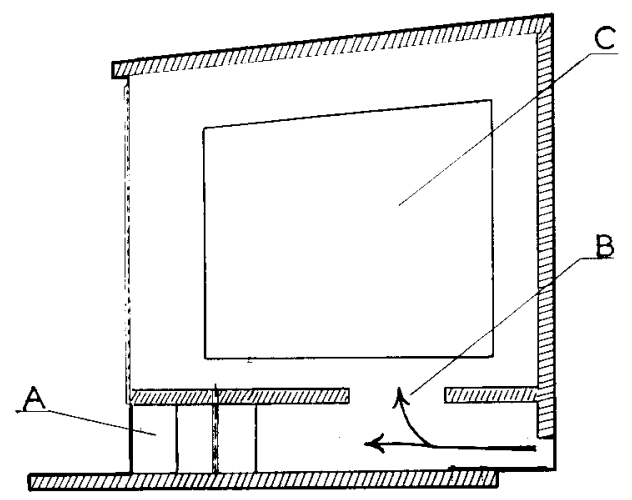

Hig. '́. - La petite cage fixée levant la ruche (coupe)

A - Entrée et sortie des abeilles

B - Orifice pour la sortie des mâles dans la cage

C - Cage de vol pour les mâles 
d'insémination artificielle. Ėlles ne sont apportées sur ces lieux qu'au fur et à mesure des besoins et $\mathrm{du}$ développement du programme de travail. Ces ruches doivent donc rester en attente dans des ruchers plus éloignés. Or, en cours de saison, les mâles naissent et dérivent. Si l'on veut éviter la destruction obligatoire de tous les mâles présents dans la ruche, seule garantie de l'absence de tout mâle étranger, il faut empêcher ceux-ci d'y pénétrer. Pour cela, on peut utiliser une grille spéciale à mâles, ou une grille à reine, mais il s'ensuivra une importante mortalité des mâles derrière la grille et il faudra la nettoyer fréquemment. Nous utilisons, de préférence, notre cage $n^{0} 3$, facile à transporter et à installer en tous lieux et que nous pouvons placer avant l'apparition des premiers mâles. Puis, deux semaines avant la période d'insémination artificielle, des ruches sont apportées près des lieux de l'insémination artificielle et installées dans les cages $n^{\circ}$ I dans lesquelles les mâles peuvent voler et parvenir à parfaite maturité. Ils pourront alors être prélevés sans s'échapper et sans se mélanger à ceux d'autres ruches.

\section{CONCLUSION}

La claustration des mâles d'abeilles destinés à l'insémination artificielle des reines au cours de travaux de sélection ou de génétique est une opération indispensable. Nous avons étudié différents types de cages qui permettent cette claustration tout en laissant aux abeilles butineuses une activité normale. Nos essais nous ont conduits à utiliser deux types de cages, 1'une de très petit volume utilisable dans des ruchers éloignés, l'autre dans laquelle on peut facilement faire les prélèvements et les visites et qui est utilisable sur les lieux mêmes de l'insémination artificielle. Les mâles peuvent y voler et atteindre une parfaite maturité sexuelle.

Nos observations nous ont montré que les mâles sont beaucoup moins désorientés dans ces cages que les ouvrières et qu'ils retrouvent la ruche plus facilement que celles-ci. Il est curieux de noter que malgré cette facilité de repérage des mâles qui est, sans aucun doute, en rapport avec le plus grand développement de leurs organes de vision et d'olfaction, ils sont, dans la nature, les individus les plus sujets au passage d'une ruche à l'autre. Il n'est pas impossible que cette tendance naturelle à la " dérive " soit en rapport avec ce qu'on pourrait appeler uise prévention de la consanguinité.

\section{Rę̧u pour publisation en novemtre 1963}

\section{SUMMARY}

THE CONFINEMENT OF MALE BEES TO BE USED FOR THE ARTIFICIAL INSEMINATION OF QUEENS

Confinement of male Apis mellifica was indispensable for selection and genetical work. Two types of cages were proposed : a very small one fixed in front of the hive for far distant apiaries and one which could be entered when visiting the hive. 'The bees pursued their normal activities of transporting nectar, pollen and water to the hive, while the males could fly in the cages to reach a healthy full maturity. The males could locate the hive more easily than could the workers, showing that they possessed more highly developed olfactory and visual senses. 


\section{RÉFÉRENCES BIBLIOGRAPHIQUES}

Chruuvin R., 1953. Le maintien de la ruche en milieu confiné est-il possible? Apiculteur Sect. Sci., $97(4), 25-29$.

FrESNAYE J., 1964. Un dispositif assurant la suppression totale du pillage et de l'agressivité des abeilles pendant les visites des ruches. Ann. Abeille, 7 (I). 47-53

Goetze G., I954. Wie stark verfliegen sich die Drohnen an einem grösseren Bienenstand? Leipzig. Bienenzeit. (West), 68 (9), 223-225.

Laidlaw H., 1954. Beekeeping Management for the Bee Breeder. Amer. Bee Journal (3), 92-95. 